\title{
Remote Sensing Image Fusion with a Multiresolution Directional-Oriented Image Transform Based on Gaussian Derivatives
}

\author{
Boris Escalante-Ramírez ${ }^{*}$ and Alejandra A. López-Caloca*, đ \\ "National Autonomous University of Mexico, School of Engineering, Electrical Division. Edif. \\ Bernardo Quintana, Circuito exterior, Cd. Universitaria, México, D.F., 04510. \\ "Center for Geography and Geomatics Research “Ing. Jorge L. Tamayo", A.C. \\ Contoy 137, Lomas de Padierna, México, D.F., 14240
}

\begin{abstract}
A methodology for image fusion based on the Hermite transform is presented. First, we demonstrate fusion with multispectral images from the same satellite (Landsat 7 ETM+) with different spatial resolutions. In this case we show how the proposed method can help improve spatial resolution. In the second case we show fusion with different sensor images, namely SAR and multispectral Landsat $5 \mathrm{TM}$. The fusion algorithm is based on the directional oriented Hermite transform which is an image representation model based on Gaussian derivatives that mimics some of the more important properties of human vision. The local analysis properties of the Hermite transform help fusion and noise reduction adapt to the local orientation and content of the image.
\end{abstract}

Keywords: Image fusion, Gaussian derivatives, Hermite transform, Steerable filters, Speckle reduction.

PACS: 07.05.Pj, 93.85.Bc, 93.85.Pq.

\section{INTRODUCCION}

In various applications, the benefit of fused images has been demonstrated for obtaining the most complete and accurate description of the studied area [1], [2]. Several authors have showed that, for image fusion, the wavelet transform approach offers good results [3], [4], [5], [6]. Comparison of Mallat and 'à trous' methodologies have been studied [7], [8]. Furthermore, multisensor image fusion algorithms based on intensity modulation have been proposed for SAR and multiband optical data fusion [9].

It is generally acknowledged that visual perception models must involve two major processing stages: 1) initial measurements and 2) high level interpretation. Fleet and Jepson [10] pointed out that the early measurement is a rich encoding of image structure in terms of generic properties from which structures that are more complex are easily detected and analyzed. Such measurement processes should be imageindependent and require no previous or concurrent interpretation. Unfortunately, it is not known what primitives are necessary and sufficient for interpretation or even 
identification of meaningful features. However, we know that, for image processing purposes, linear operators that exhibit special kinds of symmetries related to translation, rotation and magnification are of particular interest. A family of generic neighborhood operators fulfilling these requirements is that formed by the so-called Gaussian derivatives [11]. These operators have long been used in computer vision for feature extraction [12], [13], and are relevant in visual system modeling [14]. Formal integration of these operators is achieved in the Hermite transform introduced first by Martens [15], [16], and recently reformulated as a multiscale image representation model for local orientation analysis [17], [18].

The Hermite transform is an image representation model that mimics some of the more important properties of human visual perception, namely local orientation analysis and the Gaussian derivative model of early vision.

In the first part of this work, we present a methodology to fuse multispectral images from the same satellite (Landsat 7 ETM+) with different spatial resolutions. In this case we show how the proposed method can help improve spatial resolution.

In the second part, we fuse different sensor images, namely SAR and multispectral Landsat $5 \mathrm{TM}$, and show that in this case spatial resolution is also improved while spectral resolution is preserved. Parallel to image fusion, speckle reduction in the SAR image is achieved within the analysis-synthesis process of the fusion scheme. Both fusion and speckle-reduction algorithms are based on the detection of relevant image structures (primitives) during the analysis stage.

\section{HERMITE TRANSFORM}

\section{The Hermite Transform as an Image Representation Model}

The Hermite transform [15], [16], [17], [18] is a special case of polynomial transform. It can be regarded as an image description model. Firstly, windowing with a local function $\omega(x, y)$ takes place at several positions over the input image. Next, local information at every analysis window is expanded in terms of a family of orthogonal polynomials. The polynomials $G_{m, n-m}(x, y)$ used to approximate the windowed information are determined by the analysis window function and satisfy the orthogonal condition:

$$
\int_{-\infty}^{+\infty} \int_{-\infty}^{+\infty} \omega^{2}(x, y) G_{m, n-m}(x, y) G_{l, k-l}(x, y) d x d y=\delta_{n k} \delta_{m l}
$$

for $n, k=0, \ldots \ldots ; \infty, m=0, \quad, n$ and $l=0, \quad, k$; where $\delta_{n k}$ denotes the Kronecker function.

Psychophysical insights suggest using a Gaussian window function, which resembles the receptive field profiles of human vision, i.e.

$$
\omega(x, y)=\frac{1}{2 \pi \sigma^{2}} \exp \left(-\frac{\left(x^{2}+y^{2}\right)}{2 \sigma^{2}}\right)
$$


The Gaussian window is separable into Cartesian coordinates, it is isotropic, thus it is rotationally invariant and its derivatives are good models of some of the more important retinal and cortical cells of the human visual system [14], [22].

In the case of a Gaussian window function, the associated orthogonal polynomials are the Hermite polynomials [23]:

$$
G_{n-m, m}(x, y)=\frac{1}{\sqrt{2^{n}(n-m) ! m !}} H_{n-m}\left(\frac{x}{\sigma}\right) H_{m}\left(\frac{y}{\sigma}\right)
$$

where $H_{n}(x)$ denotes the $n$th Hermite polynomial

The original signal $L(x, y)$, where $(x, y)$ are the pixel coordinates, is multiplied by the window function $\omega(x-p, y-q)$, at positions $p, q$ that conform the sampling lattice $S$.

Through replication of the window function over the sampling lattice, a periodic weighting function is defined as $W(x, y)=\sum_{(p, q) \in S} \omega(x-p, y-q)$. This weighting function must be different from zero for all coordinates $(x, y)$, then:

$$
L(x, y)=\frac{1}{W(i, j)} \sum_{p, q \in S} L(x, y) \omega(x-p, y-q)
$$

The signal content within every window function is described as a weighted sum of polynomials $G_{m, n-m}(x, y)$ of $m$ degree in $x$ and $n-m$ in $y$. In a discrete implementation, the Gaussian window function may be approximated by the binomial window function, and in this case, its orthogonal polynomials $G_{m, n-m}(x, y)$ are known as the Krawtchouck's polynomials.

In either case, the polynomial coefficients $L_{m, n-m}(p, q)$ are calculated by convolution of the original image $L(x, y)$ with the function filter $D_{m, n-m}(x, y)=G_{m, n-m}(-x,-y) \omega^{2}(-x,-y)$ followed by subsampling at positions $(p, q)$ of the sampling lattice $S$, i.e.,

$$
L_{m, n-m}(p, q)=\int_{-\infty}^{+\infty} \int_{-\infty}^{+\infty} L(x, y) D_{m, n-m}(x-p, y-q) d x d y
$$

For the case of the Hermite transform, it can be shown [23] that the filter functions $D_{m, n-m}(x, y)$ correspond to Gaussians derivatives of order $m$ in $x$ and $n-m$ in $y$, in agreement with the Gaussian derivative model of early vision [14], [22].

The process of recovering the original image consists of interpolating the transform coefficients with the proper synthesis filters. This process is called an inverse polynomial transform and is defined by

$$
\hat{L}(x, y)=\sum_{n=0}^{\infty} \sum_{m=0}^{n} \sum_{(p, q) \in S} L_{m, n-m}(p, q) P_{m, n-m}(x-p, y-q)
$$


The synthesis filters $P_{m, n-m}(x, y)$ of order $m$ and $n-m$, are defined by $P_{m, n-m}(x, y)=\frac{G_{m, n-m}(x, y) \omega(x, y)}{W(x, y)}$ for $m=0, \ldots, n$ and $n=0, \ldots, \infty$.

\section{The steered Hermite transform}

The Hermite transform has the advantage that high energy compaction can be obtained through adaptively steering the transform [24], [25]. The term steerable filters describes a set of filters that are rotated copies of each other, and a copy of the filter in any orientation which is then constructed as a linear combination of a set of basis filters[26]. The steering property of the Hermite filters can be considered because the filters are products of polynomials with a radially symmetric window function. The $n+1$ Hermite filters of $n$ th-order form a steerable basis for each individual filter of $n$ order. Based on the steering property, the Hermite filters at each position in the image adapt to the local orientation content. This adaptability results in significant compaction.

For orientation analysis purposes, it is convenient to work with a rotational version of the Hermite transform. The polinomial coefficients can be computed through a convolution of the image with the filter functions $D_{m}(x) D_{n-m}(y)$, the properties of the filter functions are separable in spatial and polar domains, the Fourier transform of this filter functions are expressed in polar coordinates considering $\omega_{x}=\omega \cos \theta$ and $\omega_{y}=\omega \operatorname{sen} \theta$,

$$
d_{m}\left(\omega_{x}\right) d_{n-m}\left(\omega_{y}\right)=g_{m, n-m}(\theta) \cdot d_{n}(\omega)
$$

where $d_{n}(\omega)$ is the Fourier transform for each filter function and the radial frequency of the filter function of the $n$th order Gaussian derivative is given by

$$
d_{n}(\omega)=\frac{1}{\sqrt{2^{n} n !}}(-j \omega \sigma)^{n} \exp \left(-(\omega \sigma)^{2} / 4\right)
$$

and the orientation selectivity of the filter is expressed by

$$
g_{m, n-m}(\theta)=\sqrt{\left(\begin{array}{l}
n \\
m
\end{array}\right)} \cos ^{m} \theta \cdot \operatorname{sen}^{n-m} \theta
$$

In terms of orientation frequency functions, this property of the Hermite filters can be expressed by

$$
g_{m, n-m}\left(\theta-\theta_{0}\right)=\sum_{k=0}^{n} c_{m, k}^{n}\left(\theta_{0}\right) g_{n-k, k}(\theta)
$$

where $c_{m, k}^{(n)}\left(\theta_{0}\right)$ is the steering coefficient. The Hermite filter rotation at each position over the image is an adaptation to local orientation content. Figure 1 shows the directional Hermite decomposition over an image. First a Hermite transform was applied and then the coefficients of this transform were rotated towards the local estimated orientation, according to a maximum oriented energy criterion at each window position. For local 1D patterns, the steered Hermite transform provides a very 
efficient representation. This representation consists of a parameter $\theta$, indicating the orientation of the pattern, and a small number of coefficients, representing the profile of the pattern perpendicular to its orientation. For a 1D pattern with orientation $\theta$, the following relation holds:

$$
L_{n-m, m}^{\theta}= \begin{cases}\sum_{k=0}^{n} g_{n-k, k}(\theta) L_{n-k, k}, & m=0 \\ 0, & m>0\end{cases}
$$

For such a pattern, steering over $\theta$ results in a compaction of energy into the coefficients $L_{n, 0}^{\theta}$, while all other coefficients are set to zero.

The energy content can be expressed through the Hermite coefficients (Parseval Theorem) as

$$
E_{\infty}=\sum_{n=0}^{\infty} \sum_{m=0}^{n}\left[L_{n-m, m}\right]^{2}
$$

The energy up to $N$ order, $E_{N}$ is defined as the addition of all squared coefficients up to $N$ order.

The steered Hemite transform offers a way to describe 1D patterns on the basis of their orientation and profile. We can differentiate 1D energy terms and 2D energy terms. That is, for each local signal we have

$$
\begin{gathered}
E_{N}^{1 D}(\theta)=\sum_{n=1}^{N}\left[L_{n, 0}^{\theta}\right]^{2}, \\
E_{N}^{2 D}(\theta)=\sum_{n=1}^{N} \sum_{m=1}^{n}\left[L_{n-m, m}^{\theta}\right]^{2}
\end{gathered}
$$
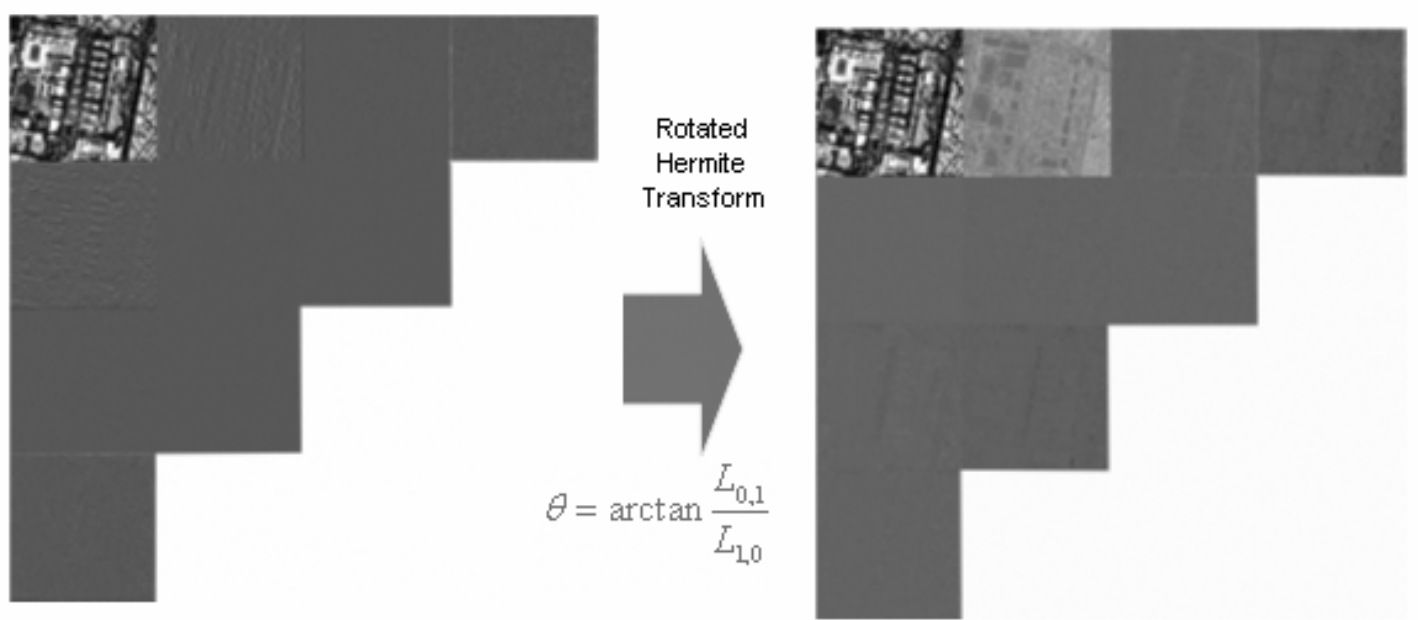

FIGURE 1. Steered Hermite Transform. Left: Original coefficients, Right: Steered coefficients. 


\section{Fusion Method Based on Hermite Transform}

The steered Hermite transform has the advantage of energy compaction. Transform coefficients are selected with an energy compaction criterion from the steered Hermite transform, therefore, it is possible to reconstruct an image with few coefficients and still preserve details such as edges and textures.

The general framework for fusion through Hermite transform includes five steps [27]: (1) Hermite transform of the image. (2) Detection of maximum energy orientation with the energy measure $E_{N}^{1 D}(\theta)$ at each window position. In practice, one estimator of the optimal orientation $\theta$, can be obtained through $\tan (\theta)=L_{0,1} / L_{1,0}$ where $L_{0,1}$ and $L_{1,0}$ are the first-order Hermite transform coefficients. (3) Adaptive steering of the transform coefficients, as described in previous sections. (4) Coefficient selection based on the method of verification of consistency [5]. This selection rule uses the maximum absolute value within a $5 \times 5$ window over the image (area of activity). The window variance is computed and used as a measurement of the activity associated with the central pixel of the window. (5) The final step of the fusion is the inverse transformation from the selected coefficients and their corresponding optimal $\theta$. Fig. 2 shows a simplified diagram of this method.

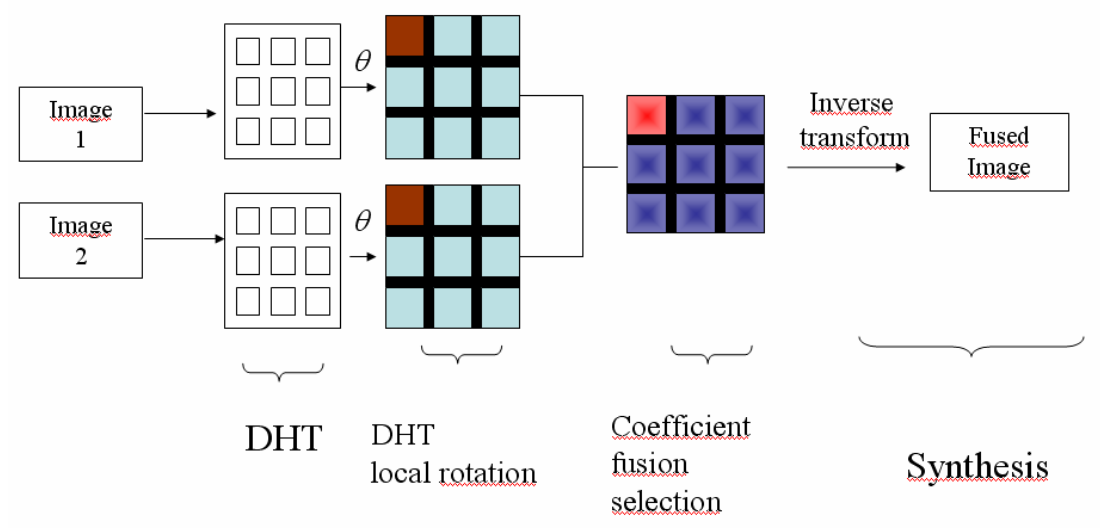

FIGURE 2. Fusion scheme with two images.

\section{Fusion Scheme with Multispectral Images and Panchromatic Images}

Our objective of image fusion is to generate synthetic images with a higher resolution that attempts to preserve the radiometric characteristics of the original multispectral data. It is desirable that any procedure that fuses high resolution panchromatic data with low resolution multispectral data preserves, as much as possible, the original spectral characteristics.

In order to apply this image fusion method, it is necessary to resample the multispectral images so that their pixel size is the same as the panchromatic image's. The steps for fusing multispectral and panchromatic images are as follows: (1) Generate new panchromatic images, whose histograms match those of each band of the multispectral image. (2) Apply the Hermite transform with local orientation 
extraction and detection of maximum energy orientation. (3) Coefficient selection based on the method of verification of consistency. (4) Inverse transformation with the optimal $\theta$ resulting from the selected coefficient set. This process of fusion is depicted in Fig. 3.

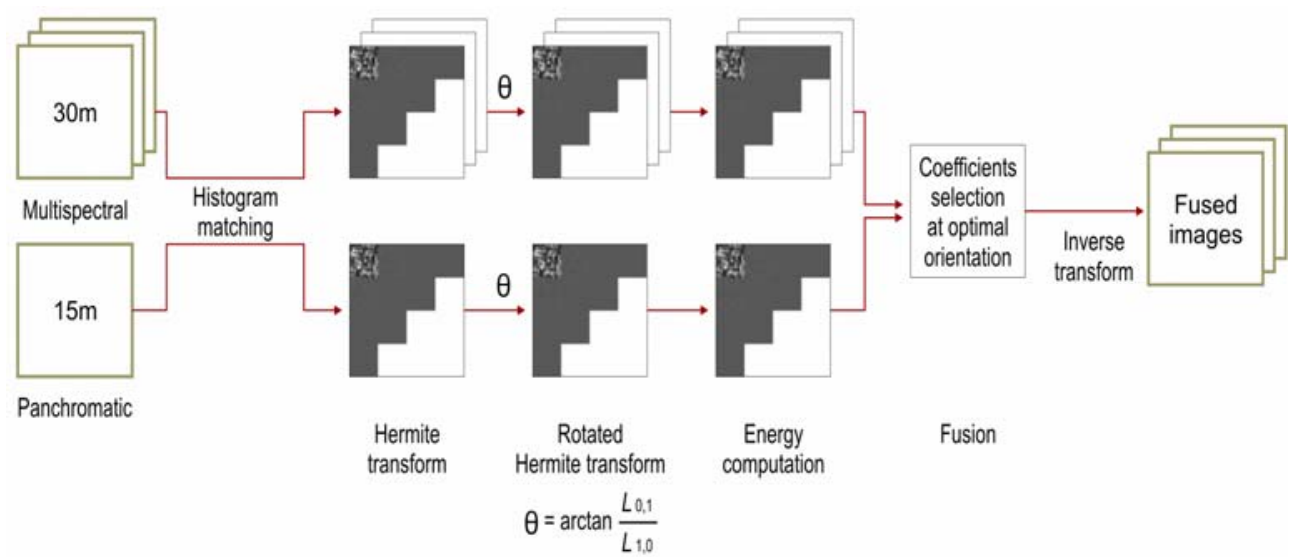

FIGURE 3. Hermite transform fusion for multispectral and panchromatic images.

The proposed fusion scheme with multispectral images has been tested on optical data. We fused multispectral images from Landsat ETM+ $(30 \mathrm{~m})$ with its panchromatic band $(15 \mathrm{~m})$. We show in figure 4 how the proposed method can help improve spatial resolution.

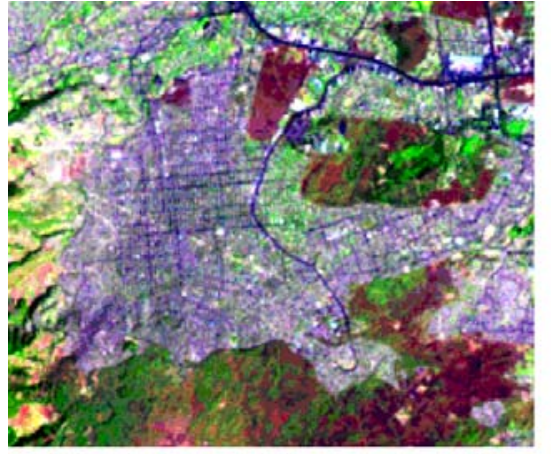

a)

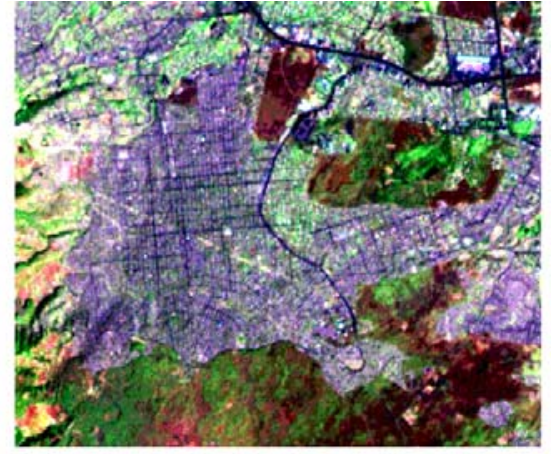

b)

FIGURE 4. (a) Original Landsat 7 ETM+ image of Mexico city (resampled to $15 \mathrm{~m}$ to match geocoded panchromatic), (b) Resulting image of ETM+ and panchromatic band fusion with Hermite Transform

(Gaussian window with spread sigma=2 and window spacing d=4, (RGB composition 5-4-3).

\section{Fusion Scheme with Multispectral Images and SAR Image}

Unfortunately, the poor quality of SAR images makes it very difficult to perform direct information extraction tasks. Numerous filters have been proposed to remove speckle in SAR imagery, however, in most cases and even in the most elegant approaches, filtering algorithms have a tendency to smooth speckle as well as 
information. We used a restoration algorithm based on the Hermite transform that adaptively smoothes images [18],[28]. Fig. 5 shows the algorithm for noise reduction.

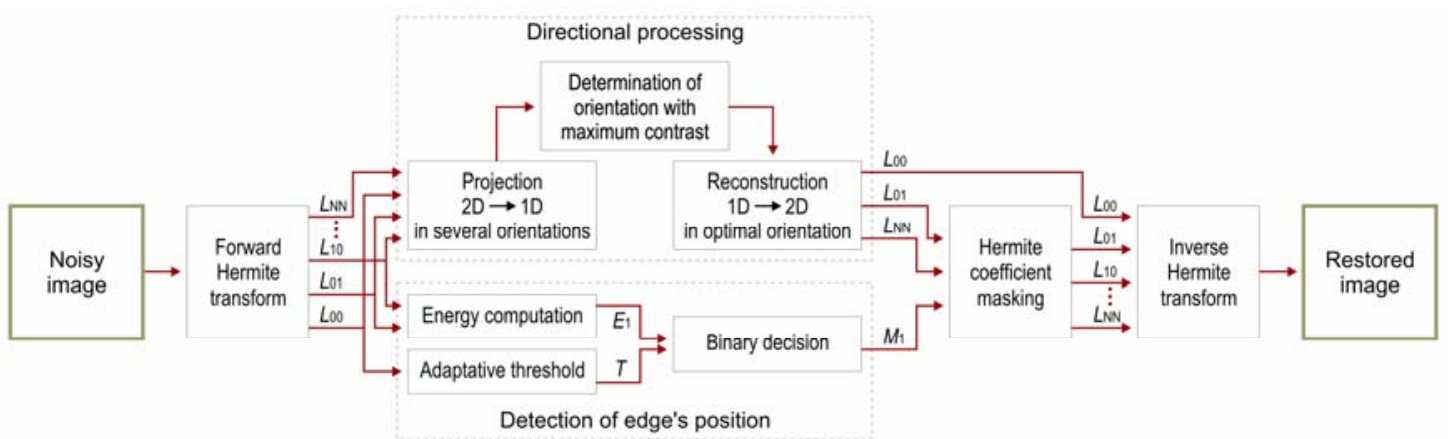

FIGURE 5. Noise-reduction algorithm.

It is easy to figure out that local orientation analysis for the purpose of noise reduction can be combined with image fusion in a single direct-inverse Hermite transform scheme. Fig. 6 shows the complete methodology to reduce noise and fuse Landsat ETM+ with SAR images. Fig. 7 illustrates the result of multispectral and SAR image HT fusion.

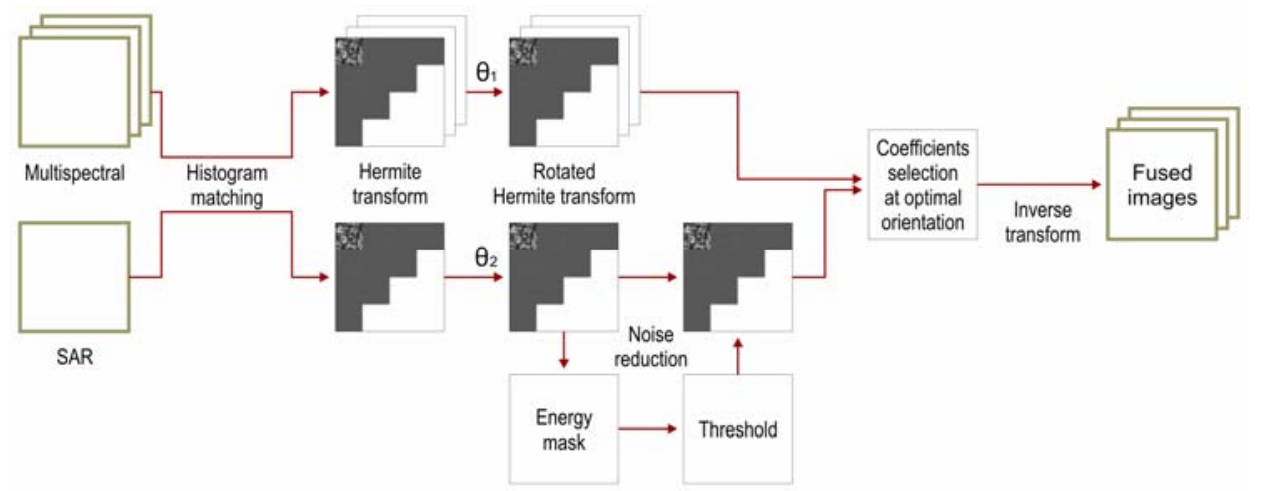

FIGURE 6. Noise-reduction and fusion for multispectral and SAR images

\section{CONCLUSIONS}

We propose a new fusion algorithm based on the discrete Hermite transform. This transform mimics some important properties of human vision, such as the Gaussian derivative model of early vision, that prove very useful for image analysis. In the specific case of image fusion, the method shows to be efficient to preserve relevant image structures. Spatial resolution is improved while spectral information is preserved. In the case of multispectral and SAR image fusion, the proposed method shows that the steering properties of the Hermite transform help reduce significantly speckle on oriented patterns. Therefore, spatial resolution is improved while speckle is limited from integrating into the fused image. 

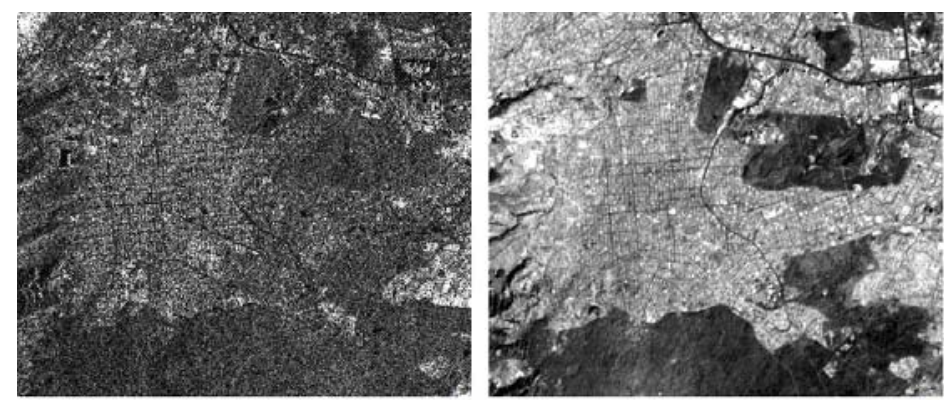

a)

b)

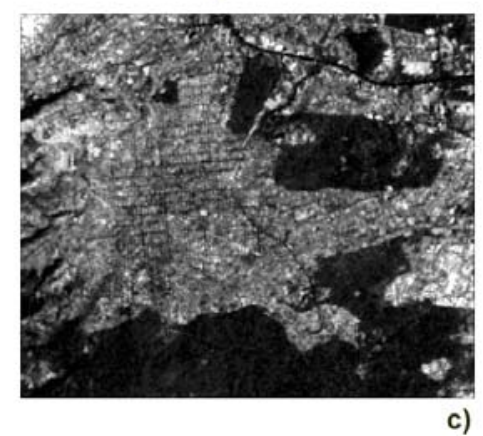

c)

FIGURE 7. a) Radarsat image with speckle (1998). b) Panchromatic Landsat-7 ETM+ (1998). c) Resulting image fusion with noise reduction..

\section{ACKNOWLEDGMENTS}

This work was sponsored by the Center for Geography and Geomatics Research "Ing.

Jorge L. Tamayo", and UNAM PAPIIT grant IN105505.

\section{REFERENCES}

1. C. Pohl, J.L. Van Genderen, "Multisensor image fusion in remote sensing: concepts, methods and applications,” International Journal of Remote Sensing, Vol 19, No, 5, pp. 823-854, 1998.

2. L. Wald, Data fusion definitions and architectures, edited by École des Mines de Paris, Paris, 2002.

3. T. Ranchin and L. Wald, "Fusion of high spatial and spectral resolution images: The ARSIS concepts and its implementation,” Photogramm. Eng. Remote Sens., vol. 66, no. 1, pp. 49-61, 2000.

4. J. Núñez, X.Otazu, O. Fors, A. Prades, V.Palà, and R. Arbiol, "Multiresolution-based image fusion with additive wavelet decomposition,” IEEE Trans. Geosci. Remote Sensing, vol. 37, no. 3, pp. 1204-1211, 1999.

5. H. Li, B. S. Manjunath, and S. K. Mitra, "Multisensor image fusion using the wavelet transform," Graphical Models and Image Processing, vol. 57, no 3, 235-245, 1995.

6. D.A. Yocky, "Image merging and data fusion by means of the discrete two-dimensional wavelet transform,” Journal of the Optical Society of America, Vol. 12, pp. 1834-1841, 1995. 
7. M. González-Audícana , J. L. Saleta, R. García Catalán and R. García, "Fusion of multispectral and panchromatic images using improved IHS and PCA mergers base don Wavelet decomposition," IEEE Trans. Geosci. Remote Sensing, vol. 42, no. 6, pp. 1291-1299, 2004.

8. Z. Wang, D. Ziou, C. Armenakis, D. Li, and Q. Li. "A comparative Analysis of Image Fusion Methods,” IEEE Trans. Geosci. Remote Sensing, vol. 43, no. 6, pp. 1391-1402, 2005.

9. L. Alparone, S. Baronti, A. Garzelli, F. Nencini, "Landsat ETM+ and SAR Image fusion based on generalized Intensity Modulation”, IEEE Trans. Geosci. Remote Sensing, vol. 42, no. 12, pp.28322839, 2004.

10. D. J. Fleet, and A. D. Jepson, "Hierarchical construction of orientation and velocity selective filters," IEEE Transactions on Pattern Analysis and Machine Intelligence Vol. II(3), pp. 315-325, 1989.

11. J. Koenderink, and A. J. Van Doorn, "Generic neighborhood operators," IEEE Transactions on Pattern Analysis and Machine Intelligence. Vol. 14, pp. 597-605, 1992.

12. J. Bevington, and R. Mersereau, "Differential operator based edge and line detection," in Proceedings ICASSP, pp. 249-252, 1987.

13. V. Torre, and T. Poggio, "On edge detection," IEEE Transactions on Pattern Analysis and Machine Intelligence. Vol. 8, pp. 147-163, 1986.

14. R. Young, "The Gaussian derivative theory of spatial vision: analysis of cortical cell receptive field line-weighting profiles,” General Motors Res. Labs., Rep. 4920, 1986.

15. J. B. Martens, "The Hermite Transform -Theory," IEEE Transactions on Acoustics, Speech and Signal processing, vol. 38, no. 9, pp. 1607-1618, 1990.

16. J. B. Martens, “The Hermite Transform -Aplications,” IEEE Transactions on Acoustics, Speech and Signal Processing, vol. 38, No. 9, 1595-1606, 1990.

17. J.L. Silván-Cárdenas, B. Escalante-Ramírez, "The multiscale Hermite transform for local orientation analysis," IEEE Transactions on Image Processing, vol. 15, No. 5, pp.1236-1253, 2006.

18. B. Escalante-Ramírez, J.L. Silván-Cárdenas, “Advanced modeling of visual information processing: A multirresolution directional-oriented image transform based on Gaussian derivatives,” Signal Process: Image Communication, vol. 20, No. 9-10, pp. 801-812, 2005

19. E. P. Crist, R. C. Cicone, "A physically-based transformation of Thematic Mapper data-the -TM Tasselep Cap,” IEEE Trans. Geosci. Remote Sensing, GE-22, no. 3, pp. 256-263, 1984.

20. E. P. Crist, R. J. Kauth, "The tasseled cap de-mystified," Photogrammetric Engineering and Remote sensing, vol.52, no.1, pp. 81-86, 1986.

21. E. P. Crist, R. C. Cicone, "Application of the Tasseled Cap concept to simulated Thematic Mapper data," Photogrammetric Engineering and Remote Sensing, vol. 50, no.3, pp. 343-352, 1984.

22. R. Young, "Oh say, can you see?. The physiology of vision," Proc. SPIE, Vol. 1453, pp. 92-723, 1991.

23. G. Szegö, Orthogonal Polynomials, American Mathematical Society, Colloquium Publications, 1959.

24. J. B. Martens, "Local Orientation Analysis in images by means of the Hermite Transform," IEEE Transactions on Image Processing, vol. 6, No. 8, pp. 1103-1116, 1997.

25. A. M. Van Dijk, J. B. Martens, "Image representation and compression with steered Hermite transform,” Signal Processing, vol. 56, pp. 1-16, 1997.

26. W.T. Freeman, E.H. Adelson, "The design and use of steerable filters," IEEE Transactions on Pattern Analysis and Machine Intelligence, Vol. 13 (9), pp. 891-906, 1991.

27. B. Escalante-Ramírez, A. López-Caloca, "Image fusion with the Hermite Transform," in Conf. ICIP 2003, International conference on Image Processing 14th ,Barcelona, Spain.

28. B. Escalante-Ramírez, J. B. Martens, "Noise Reduction in Computerized Tomography Images by Means of Polynomial Transforms," Journal of visual Communication and Image Representation., vol. 3, no. 3, pp. 272-285, 1992. 